\title{
Acute Effect of Sodium Nitroprusside on Microvascular Dysfunction in Patients Who Underwent Percutaneous Coronary Intervention for Acute ST-segment Elevation Myocardial Infarction
}

\author{
Kotaro MоRimoto, ${ }^{1,2}$ MD, Shigenori Ito, ${ }^{2}$ MD, Kosuke NAKasuka, ${ }^{2}$ MD, Satoru SeKimoto, ${ }^{2}$ MD, \\ Kazuyuki MiYATA, ${ }^{2} \mathrm{MD}$, Masahiko Inomata, ${ }^{2} \mathrm{MD}$, Takayuki Yoshida, ${ }^{2} \mathrm{MD}$, Nozomu TAmaI, ${ }^{2} \mathrm{MD}$, \\ Tomoaki SAEKI, ${ }^{2}$ MD, Shin Suzuki, ${ }^{2}$ MD, Yoshimasa Murakami, ${ }^{2}$ MD, Koichi SATo, ${ }^{2}$ MD, \\ Akihiro Morino, ${ }^{3} \mathrm{CE}$, and Yoshiyuki Shimizu, ${ }^{3} \mathrm{CE}$
}

\begin{abstract}
SUMMARY
Even in the era of thrombus aspiration and distal protection for ST-segment elevation acute myocardial infarction (STEMI), microvascular dysfunction does exist and improvement of microvascular dysfunction can improve the prognosis and/or left ventricular dysfunction. We evaluated the acute effects of nitroprusside (NTP) on coronary microvascular injury that occurred after primary percutaneous coronary intervention (PCI) for STEMI in 18 patients. The final Thrombolysis in Myocardial Infarction trial (TIMI) flow grade after PCI was 3 in 17 patients and 2 in 1 patient. The index of microcirculatory resistance (IMR) was improved significantly from $76 \pm 42$ to $45 \pm 37(P=0.0006)$ by intracoronary NTP administration. IMR improved to the normal range (IMR < 30) in 9 patients $(50 \%)$. Higher TIMI flow grade and lower IMR at baseline were observed more frequently in patients whose IMR recovered to normal range after NTP administration. NTP improved the microcirculatory dysfunction at the acute phase in patients who underwent PCI for STEMI and had final TIMI 3 flow in almost all cases. (Int Heart J 2012; 53: 337-340)
\end{abstract}

Key words: Acute myocardial infarction, Reperfusion, Index of microcirculatory resistance, Slow flow, Complication

$\mathrm{S}$ everal methods have been proposed to evaluate the prognosis of patients with acute myocardial infarction (AMI) who underwent percutaneous coronary intervention (PCI). Despite the importance of microvascular coronary dysfunction for a prognosis, accurate estimation of microvascular function is challenging immediately after reperfusion for AMI. Although epicardial coronary blood flow at final angiography is one of the predictive factors with which to evaluate left ventricular function recovery in the chronic stage, thrombolysis in myocardial infarction trial (TIMI) flow grade 3 is not adequate and more accurate modalities have been reported, such as the corrected TIMI flow count ${ }^{1)}$ and Blush score. ${ }^{2)}$ Fearon, et $a l^{3)}$ reported that, compared to other traditional methods for assessing the microcirculation, the index of microcirculatory resistance (IMR) is a better predictor of microvascular damage and recovery of left ventricular function after STsegment elevation AMI (STEMI). Sezer, et $a l^{4)}$ also reported that the IMR measured early (2 days) after STEMI independently predicted the infarct size at follow-up (5 months). Treatment for microcirculation injury after PCI for an occluded coronary artery is important, although evidence showing its effect is scarce. On the basis of these findings, further studies evaluating therapeutic approaches for improving microvascular integrity at the acute phase of STEMI that might improve infarct healing and functional recovery are warranted. Sodium nitro- prusside (NTP), which is a direct donor of nitric oxide, ${ }^{5)}$ strongly dilates the resistance arterioles and plays a significant role in coronary blood flow through the microcirculation, ${ }^{6,7)}$ and requires no intracellular metabolism to derive nitric oxide. Thus, this study was planned to evaluate the effect of intracoronary administration of NTP on impaired microcirculation in patients who underwent PCI for STEMI.

\section{Methods}

Study subjects: The subjects included patients who underwent PCI for STEMI and had suspected microvascular dysfunction, defined as a final IMR $\geqq 30$ after PCI, and who were treated with intracoronary administration of NTP between August 2010 and June 2011. The diagnosis of STEMI was made on the basis of chest pain lasting for more than 30 minutes and 0.1 $\mathrm{mV}$ ST-segment elevation in 2 continuous ECG leads and CPK elevation more than 3 times the normal range. Time from symptom onset to admission was less than 24 hours in all cases. The study was approved by the Ethics Committee of our hospital. All patients provided informed written consent. Antiplatelet therapy was performed with a loading dose of 300 $\mathrm{mg}$ of clopidogrel, 100 or $200 \mathrm{mg}$ of aspirin, and $200 \mathrm{mg}$ of cilostazol before emergency PCI if not prescribed before ad-

From the ${ }^{1}$ Division of Cardiology, Asahi Rosai Hospital, ${ }^{2}$ Division of Cardiology, Nagoya City East Medical Center, and ${ }^{3}$ Division of Clinical Engineering, Nagoya City East Medical Center, Aichi, Japan.

Address for correspondence: Shigenori Ito, MD, Division of Cardiology, Nagoya City East Medical Center, 1-2-23 Wakamizu-cho, Chikusa-ku, Nagoya, Aichi 4648547, Japan.

Received for publication May 14, 2012

Revised and accepted August 20, 2012. 
mission. Prior to coronary angiography, all patients received 3,000 $\mathrm{U}$ of intravenous heparin and then 7,000 U before PCI. Intracoronary isosorbide dinitrate (ISDN) was also given before the control and final angiographies, and at other times if needed.

PCI procedure: A 6 French guiding catheter without side holes was inserted via a transradial approach. After control coronary angiograms and a diagnosis of STEMI, thrombus aspiration was usually performed several times and distal protection with Filtrap ${ }^{\mathrm{TM}}$ (Nipro, Osaka, Japan) was also performed based on the IVUS and OCT findings at the discretion of the operator.

IMR measurements and administration of NTP: After successful stenting of the culprit lesion, final coronary angiograms were taken after intracoronary injection of ISDN (1-3 mg). Then, IMR was measured under hyperemia induced by intravenous adenosine triphosphate $(150 \mu \mathrm{g} / \mathrm{kg} /$ minute for $2-3$ minutes) after the final coronary angiogram. When IMR was $\geqq 30$, NTP $(100 \mu \mathrm{g})$ diluted with $10 \mathrm{~mL}$ of saline was administered into the culprit coronary artery and IMR was measured again. A PressureWire (St. Jude Medical, MN, USA) was carefully placed in the distal two-thirds of the coronary artery, which was beyond the stented region, at the identical location at baseline and after NTP administration. The position of the sensor was recorded by angiography during baseline measurements as a point of reference. Adenosine 5'-triphospate $(0.15$ $\mathrm{mg} \cdot \mathrm{kg}-1 \cdot \mathrm{minute}-1)$ was injected intravenously. The hyperemic mean transit time was then measured and averaged after 3 injections of $3 \mathrm{~mL}$ of room temperature saline through the guiding catheter as previously described. ${ }^{8)}$

Coronary risk factors: Coronary risk factors were determined as follows: diabetes mellitus, a history or the presence of dia-

Table I. Baseline Patient and Angiographic Characteristics

\begin{tabular}{lc}
\hline Number of patients & 18 \\
Age (years) & $75.5 \pm 42.0(38-93)$ \\
Male gender & $12(66.7 \%)$ \\
Hypertension & $12(66.7 \%)$ \\
Diabetes mellitus & $4(22.2 \%)$ \\
Dyslipidemia & $11(61.1 \%)$ \\
Smoking (current/former/never) & $8 / 3 / 7$ \\
& \\
Angina prior to infarction & $8(44.4 \%)$ \\
Killip classification (I/II/III/IV) & $9 / 6 / 1 / 2$ \\
Onset-to-balloon time (hours) & $7.8 \pm 8.0$ \\
Door-to-balloon time (minutes) & $91 \pm 48$ \\
& \\
Aspiration & $17(94.4 \%)$ \\
Use of Filtrap & $10(55.6 \%)$ \\
Filter no-reflow & $6 / 10(60 \%)$ \\
Max CPK (IU/L) & $2858 \pm 2148(490-6881)$ \\
Use of nicorandil & 13 \\
Number of diseased vessels (1/2/3) & \\
Infarcted artery (LAD/LCx/RCA) & $8 / 7 / 3$ \\
Rentrop collateral grade (0/1/2/3) & $7 / 3 / 8$ \\
Pre-TIMI grade (0/1/2) & $8 / 9 / 1 / 0$ \\
Final TIMI grade (2/3) & $9 / 2 / 7$ \\
\hline
\end{tabular}

CPK indicates creatine phosphokinase; LAD, left anterior descending artery; LCx, left circumflex artery; RCA, right coronary artery; and TIMI, thrombolysis in myocardial infarction. betes and/or a fasting plasma glucose concentration $>126 \mathrm{mg} /$ $\mathrm{dL}$ and/or a glycosylated hemoglobin level $\geqq 6.5 \%$; hypertension, a history of hypertension and/or systolic blood pressure $\geqq 140 \mathrm{mmHg}$ and/or diastolic pressure $\geqq 90 \mathrm{mmHg}$; dyslipidemia, a history of dyslipidemia and/or LDL cholesterol $\geqq 140 \mathrm{mg} / \mathrm{dL}$ and/or HDL cholesterol $<40 \mathrm{mg} / \mathrm{dL}$ and/or triglycerides $\geqq 150 \mathrm{mg} / \mathrm{dL}$.

Statistical analysis: Values are expressed as the mean \pm standard deviation. The Wilcoxon signed-rank test was used for paired comparisons of continuous values. The unpaired $t$-test or the Mann-Whitney test was used for unpaired comparisons. Fisher's exact test or the chi-square test was used to examine differences between categorical variables. $P$ values $<0.05$ were considered significant. Statistical analysis was performed using Stat View version 5.0 (SAS Institute, Cary, NC, USA).

\section{RESULTS}

Baseline patient and angiographic characteristics: IMR was measured in a total of 19 STEMI patients and was $\geqq 30$ in all 19 patients during the study period. Eighteen patients were included in the study. NTP could not be administered in one patient. Table I shows the baseline clinical and angiographic characteristics. Mean age was $75.5 \pm 42.0$ years and $66.7 \%$ $(12 / 18)$ were male. Target vessels were the right coronary artery in 8 , left anterior descending artery in 7, and left circumflex artery in 3. Coronary risk factors are shown in Table I. PreTIMI flow grade was 0 in 9, 1 in 2, and 2 in 7 patients. Aspiration was performed in 17 patients $(94.4 \%)$. A Filtrap ${ }^{\mathrm{TM}}$ was used in 10 patients $(55.6 \%)$. Filter no reflow occurred in 6 patients $(60 \%)$ during the procedure. TIMI 3 flow grade at the final angiogram was obtained in 17 patients $(94.4 \%)$. Nicorandil was administered by intracoronary injection before reperfusion and continued intravenously during PCI in 12 patients $(66.7 \%)$, at the discretion of the operator. Pre-TIMI flow grade was 0 in 2, 1 in 1,2 in 2, and 3 in 1 patient among 6 patients who were not given nicorandil.

Change in IMR after NTP administration: IMR improved significantly from $76 \pm 42$ to $45 \pm 37(P=0.0006)$ by NTP administration in the 18 patients (Figure). In 1 patient with a final TIMI flow of grade 2, IMR increased from 128 to 141 with distal embolism in a side branch. In another patient IMR increased from 35 to 46 . Onset to balloon time was 23 hours in

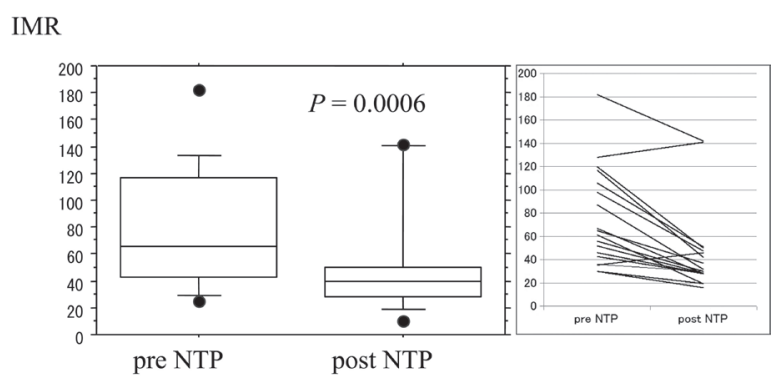

Figure. Change in IMR between before and after NTP administration. IMR was significantly decreased after NTP administration in 18 patients. IMR was not decreased in only 2 cases. IMR indicates index of microcirculatory resistance and NTP, nitroprusside. 
Table II. Comparison of Clinical Factors Between Patients With IMR $\geqq$ 30 and IMR $<30$ After NTP

\begin{tabular}{|c|c|c|c|}
\hline & $\begin{array}{c}\text { IMR } \geqq 30 \\
\text { after NTP } \\
n=9\end{array}$ & $\begin{array}{c}\mathrm{IMR}<30 \\
\text { after NTP } \\
n=9\end{array}$ & $P$ \\
\hline Age (years) & $66.3 \pm 13.6$ & $70.6 \pm 16.6$ & 0.563 \\
\hline Male/Female & $6 / 3$ & $6 / 3$ & 1.000 \\
\hline Hypertension & 5 & 7 & 0.620 \\
\hline Diabetes mellitus & 2 & 2 & 1.000 \\
\hline Dyslipidemia & 5 & 6 & 1.000 \\
\hline Smoking (current/former/never) & $3 / 5 / 1$ & $4 / 3 / 2$ & 0.614 \\
\hline Angina prior to infarction & 5 & 3 & 0.637 \\
\hline Killip classification (1/2/3/4) & $4 / 2 / 1 / 2$ & $5 / 4 / 0 / 0$ & 0.287 \\
\hline Onset-to-balloon time (hours) & $10.2 \pm 3.7$ & $5.5 \pm 5.5$ & 0.218 \\
\hline Door-to-balloon time (minutes) & $104 \pm 57$ & $78 \pm 35$ & 0.263 \\
\hline Aspiration & 9 & 8 & 1.000 \\
\hline Use of Filtrap & 4 & 6 & 0.637 \\
\hline Max CPK (IU/L) & $3356.2 \pm 2280.3$ & $2359.6 \pm 2011.4$ & 0.340 \\
\hline Use of nicorandil & 6 & 7 & 1.000 \\
\hline $\begin{array}{l}\text { Number of diseased vessels } \\
(1 \mathrm{VD} / 2 \mathrm{VD} / 3 \mathrm{VD})\end{array}$ & $4 / 3 / 2$ & $4 / 4 / 1$ & 0.788 \\
\hline Vessel (LAD/LCx/RCA) & $2 / 1 / 6$ & $5 / 2 / 2$ & 0.164 \\
\hline $\begin{array}{l}\text { Rentrop collateral grade } \\
(0 / 1 / 2 / 3)\end{array}$ & $3 / 5 / 1 / 0$ & $5 / 4 / 0 / 0$ & 0.447 \\
\hline Pre TIMI grade (0/1/2) & $7 / 1 / 1$ & $2 / 1 / 6$ & 0.042 \\
\hline Final TIMI grade (2/3) & $1 / 8$ & $0 / 9$ & 1.000 \\
\hline Pre IMR & $104.3 \pm 46.7$ & $46.7 \pm 13.5$ & 0.001 \\
\hline IMR after NTP & $65.4 \pm 43.6$ & $25.2 \pm 5.4$ & 0.015 \\
\hline
\end{tabular}

IMR indicates index of microcirculatory resistance; NTP, nitroprusside; VD, vessels diseased; LAD, left anterior descending artery; LCx, left circumflex artery; RCA, right coronary artery; TIMI, thrombolysis in myocardial infarction; and CPK, creatine phosphokinase.

this patient. Both patients had large max CPK over 4000 (IU/L) due to right coronary artery occlusion. In 9 patients (50\%), IMR improved to within the normal range (IMR $<30$ ). TIMI flow grade and IMR at baseline were related to the recovery of IMR to the normal range after PCI (Table II). IMR at baseline, IMR after NTP administration, and change in IMR were not significantly different between patients with and without administration of nicorandil (Table III).

\section{Discussion}

In this study, it was confirmed that microvascular coronary dysfunction occurs even in patients with TIMI 3 flow after reperfusion using IMR. We also showed that NTP could improve IMR in patients with STEMI, even in patients with TIMI 3 flow but a high IMR after primary PCI for STEMI.

Previously, IMR after reperfusion was shown to predict left ventricular infarct size and recovery at follow-up in patients with STEMI who underwent primary PCI., ${ }^{3,4}$ Thus, the improvement of IMR at acute phase is expected to improve LV function. The normal range of IMR in humans has not yet been determined. Fearon, et $a l^{3)}$ reported that the IMR was the only significant predictor of recovery of left ventricular function based on the percent change in wall motion score evaluated by
Table III. Comparison of Pre IMR, Post IMR, and Change in IMR Between Patients With and Without Nicorandil

\begin{tabular}{lccc} 
& $\begin{array}{c}\text { Nicorandil (+) } \\
n=12\end{array}$ & $\begin{array}{c}\text { Nicorandil (-) } \\
n=6\end{array}$ & $P$ \\
\hline Pre IMR & $76.1 \pm 46.5$ & $74.3 \pm 32.1$ & 0.934 \\
Post IMR & $49.3 \pm 43.9$ & $37.4 \pm 13.3$ & 0.532 \\
Change in IMR (Pre-Post) & $26.9 \pm 24.5$ & $36.9 \pm 27.7$ & 0.402 \\
\hline
\end{tabular}

IMR indicates index of microcirculatory resistance.

echocardiography. In patients with an IMR $\leqq 32$, the wall motion score improved significantly from $25.4 \pm 6.6$ at baseline to $19.5 \pm 3.6(P=0.0002)$ at 3 months. In patients with an IMR $>32 \mathrm{U}$, the wall motion score did not change significantly. Based on this report, we defined microvascular dysfunction, which is associated with a low probability of left ventricular function recovery, as a final IMR $\geqq 30$ after primary PCI to evaluate only patients with suspected microvascular dysfunction after primary PCI.

Recently, Ito, et $a l^{9)}$ reported the effect of intracoronary administration of nicorandil, which is a vasodilator that works both as a nitrate and a K-ATP channel activator, on IMR decrease during PCI. Kobatake, et $a l^{10)}$ compared the effects of NTP versus nicorandil on the slow/no-reflow phenomenon during coronary intervention for acute myocardial infarction and concluded that NTP is a more effective treatment for slow/ no-reflow using the TIMI frame count $(37.8 \pm 15.1$ to $13.7 \pm$ 7.1 in NTP versus $30.8 \pm 20.7$ to $19.3 \pm 17.9$ in nicorandil) and TIMI flow grade $(1.64 \pm 0.62$ to $2.74 \pm 0.36$ in NTP versus $1.60 \pm 0.86$ to $2.23 \pm 0.91$ in nicorandil). ${ }^{10)}$ In our study, 12 patients received nicorandil prior to NTP administration, and there was no difference in the incidence of recovery to a normal IMR level and change in IMR after NTP administration between patients with and without nicorandil.

Sodium nitroprusside (NTP) is not a novel drug that had been used to decrease blood pressure. Recently, some believe it may be a direct donor of nitric oxide, ${ }^{5)}$ which strongly dilates the resistance arterioles and plays a significant role in coronary blood flow through the microcirculation, ${ }^{6,7)}$ and requires no intracellular metabolism to produce nitric oxide. It has been shown that intracoronary NTP injection is a safe and effective technique for managing the slow reflow phenomenon once it occurs during coronary intervention $1 .{ }^{11-14)}$ Thus, we believe that it is reasonable to use NTP for microvascular dysfunction, although we could not verify its effect is persistent after PCI in this study.

Microvascular injury can be measured with $\mathrm{IMR}^{15)}$ in addition to the TIMI frame count ${ }^{1)}$ or Blush score ${ }^{2)}$ and has been recognized as an accurate marker of microcirculatory injury. In fact, IMR measurement is a useful tool with which to evaluate microvascular injury, even in patients with TIMI $^{3)}$ flow after PCI for STEMI, and the effects of drugs. Furthermore, this method may be more quantitative than the Blush score and more physiological than corrected TIMI frame count at the expense of invasiveness.

This study has several limitations. First, it was an observational study with a small sample size and was not a randomized study with a control group. However, in a previous report $^{9)}$ it was shown that administration of $10 \mathrm{~mL}$ of saline did 
not change the IMR. Second, there are no follow-up data available which show the benefit of NTP improvement at index PCI procedure. Third, how long the improvement of IMR is maintained after administration should also be elucidated. Fourth, we could not analyze the corrected TIMI frame count according to the method of Gibson, et $a l^{16)}$ or myocardial blush grade according to the method of van'Hof, et $a l^{2)}$ as other modalities to evaluate microvascular function. However, we believe that IMR is already recognized as a validated tool. Finally, IMR increased in two patients in this study despite the administration of nitroprusside. In the first patient, the final TIMI flow was 2 and there was a distal embolism in a side branch. In the second case, onset to balloon time was 23 hours. Both patients had large max CPK over 4000 due to right coronary artery occlusion. However, we were unable to determine why the IMR worsened. A larger study is warranted to elucidate the unresponsiveness to IMR.

Conclusion: NTP improved the microcirculatory dysfunction at the acute phase in almost all patients who underwent primary PCI for STEMI and had final TIMI 3 flow.

\section{REFERENCES}

1. Zalewski J, Zmudka K, Musialek P, et al. Detection of microvascular injury by evaluating epicardial blood flow in early reperfusion following primary angioplasty. Int J Cardiol 2004; 96: 38996.

2. van't Hof AW, Liem A, Suryapranata H, Hoorntje JC, de Boer MJ, Zijlstra F. Angiographic assessment of myocardial reperfusion in patients treated with primary angioplasty for acute myocardial infarction: myocardial blush grade. Zwolle Myocardial Infarction Study Group. Circulation 1998; 97: 2302-6.

3. Fearon WF, Shah M, Ng M, et al. Predictive value of the index of microcirculatory resistance in patients with ST-segment elevation myocardial infarction. J Am Coll Cardiol 2008; 51: 560-5

4. Sezer M, Aslanger EK, Cimen AO, et al. Concurrent microvascular and infarct remodeling after successful reperfusion of ST-elevation acute myocardial infarction. Circ Cardiovasc Interv 2010; 3: 208-15.

5. Bates JN, Baker MT, Guerra R Jr, Harrison DG. Nitric oxide generation from nitroprusside by vascular tissue. Evidence that reduc- tion of the nitroprusside anion and cyanide loss are required. Biochem Pharmacol 1991; 42 Suppl: S157-65.

6. Kuo L, Chilian WM, Davis MJ. Interaction of pressure- and flowinduced responses in porcine coronary resistance vessels. Am J Physiol 1991; 261: H1706-15.

7. Myers PR, Banitt PF, Guerra R Jr, Harrison DG. Role of the endothelium in modulation of the acetylcholine vasoconstrictor response in porcine coronary microvessels. Cardiovasc Res 1991; 25: 129-37.

8. De Bruyne B, Pijls NH, Smith L, Wievegg M, Heyndrickx GR. Coronary thermodilution to assess flow reserve: experimental validation. Circulation 2001; 104: 2003-6.

9. Ito N, Nanto S, Doi Y, et al. High index of microcirculatory resistance level after successful primary percutaneous coronary intervention can be improved by intracoronary administration of nicorandil. Circ J 2010; 74: 909-15.

10. Kobatake R, Sato T, Fujiwara Y, et al. Comparison of the effects of nitroprusside versus nicorandil on the slow/no-reflow phenomenon during coronary interventions for acute myocardial infarction. Heart Vessels 2011; 26: 379-84.

11. Hillegass WB, Dean NA, Liao L, Rhinehart RG, Myers PR. Treatment of no-reflow and impaired flow with the nitric oxide donor nitroprusside following percutaneous coronary interventions: initial human clinical experience. J Am Coll Cardiol 2001; 37: 133543.

12. Pasceri V, Pristipino C, Pelliccia F, et al. Effects of the nitric oxide donor nitroprusside on no-reflow phenomenon during coronary interventions for acute myocardial infarction. Am J Cardiol 2005; 95: 1358-61.

13. Wang HJ, Lo PH, Lin JJ, Lee H, Hung JS. Treatment of slow/noreflow phenomenon with intracoronary nitroprusside injection in primary coronary intervention for acute myocardial infarction. Catheter Cardiovasc Interv 2004; 63: 171-6.

14. Shinozaki N, Ichinose H, Yahikozawa K, Shimada H, Hoshino K. Selective intracoronary administration of nitroprusside before balloon dilatation prevents slow reflow during percutaneous coronary intervention in patients with acute myocardial infarction. Int Heart J 2007; 48: 423-33.

15. Fearon WF, Balsam LB, Farouque HM, et al. Novel index for invasively assessing the coronary microcirculation. Circulation 2003; 107: 3129-32.

16. Gibson CM, Cannon CP, Daley WL, et al. TIMI frame count: a quantitative method of assessing coronary artery flow. Circulation 1996; 93: 879-88. 